\title{
Ginsenoside Rd in Experimental Stroke: Superior Neuroprotective Efficacy with a Wide Therapeutic Window
}

\author{
Ruidong Ye • Xiangwei Kong • Qianzi Yang • \\ Yunxia Zhang • Junliang Han • Ping Li • Lize Xiong • \\ Gang Zhao
}

Published online: 7 June 2011

(C) The American Society for Experimental NeuroTherapeutics, Inc. 2011

\begin{abstract}
Ginsenoside $\mathrm{Rd}(\mathrm{Rd})$, one of the main active ingredients in Panax ginseng, has been demonstrated to protect against ischemic cerebral damage in vitro and in vivo. In this study, we aimed to further define the preclinical characteristics of Rd. We show that Rd passes the intact blood-brain barrier and exerts protection in both transient and permanent middle cerebral artery occlusion (MCAO) in rats. In the dose-response study, $\mathrm{Rd}(10-50 \mathrm{mg} / \mathrm{Kg})$ significantly reduced the infarct volume on postoperative days (PODs) 1, 3, and 7. This protection was associated with an improved neurological outcome for as many as 6 weeks after transient MCAO, as assessed by modified neurological severity score, modified sticky-tape test, and corner test. For comparison, Rd was significantly more effective than edaravone and slightly more effective than Ntert-butyl-alpha-phenylnitrone (PBN). In the therapeutic
\end{abstract}

Ruidong Ye, Xiangwei Kong and Qianzi Yang contributed equally to this work.

Electronic supplementary material The online version of this article (doi:10.1007/s13311-011-0051-3) contains supplementary material, which is available to authorized users.

R. Ye $\cdot$ Y. Zhang $\cdot$ J. Han $\cdot$ G. Zhao $(\bowtie)$

Department of Neurology, Xijing Hospital,

Xi'an, China

e-mail: zhaogang@fmmu.edu.cn

Q. Yang $\cdot$ L. Xiong

Department of Anesthesiology, Xijing Hospital,

Xi'an, China

P. Li

Department of Orthopedics, Xijing Hospital,

Xi'an, China

X. Kong

College of Stomatology, the Fourth Military Medical University,

Xi'an, China 710032 window study, Rd exhibited remarkable neuroprotection, even when administered for as many as $4 \mathrm{~h}$ after the recirculation of transient $\mathrm{MCAO}$ or after the onset of permanent MCAO. Furthermore, in female rats or 16month-old male rats, the salutary effects of Rd were also observed. These findings suggest $\mathrm{Rd}$ is a promising neuroprotectant and provide support for future clinical studies to confirm whether Rd is beneficial in ischemic stroke.

Keywords Ginsenoside Rd · Middle cerebral artery occlusion $\cdot$ Neuroprotection $\cdot$ Stroke $\cdot$ Cerebral ischemia

\section{Introduction}

Ischemic stroke ranks among the leading causes of death and adult disability worldwide. Two major approaches have been developed to treat acute ischemic stroke: recanalization and neuroprotection. Thrombolysis with tissue plasminogen activator, limited by its narrow therapeutic time window and the concern of hemorrhagic complication, is still uncommon in use [1]. In the US, only 3 to $5 \%$ of patients with ischemic stroke receive tissue plasminogen activator [2]. The goal of neuroprotective strategies is to protect the penumbra brain tissue and attenuate clinical sequelae of stroke. The development of neuroprotective therapy for acute ischemic stroke is challenging. Although a large number of neuroprotective interventions have demonstrated efficacy in preclinical studies involving small animals, clinical studies have so far failed to demonstrate similar efficacy $[3,4]$.

Ginseng, the root of Panax ginseng C. A. Meyer (Araliaceae), has been used as a tonic to treat a wide variety of disorders in China for millennia. Extensive studies have proved that the molecular components respon- 
sible for the pharmacologic effects of ginseng are ginseng saponins, namely ginsenosides. Currently, more than 30 ginsenosides have been identified and isolated from ginseng. Dammar-24(25)-ene-3b,12b,20(S)-triol-(20-O-bD-glucopyranosyl)-3-O-b-D-glucopyranosyl-(1 $\rightarrow 2)-b-D-$ glucopyranoside (ginsenoside $\mathrm{Rd}, \mathrm{Rd}$ ) is one of the main active components of ginsenosides. Previously, we found that $\mathrm{Rd}$ could protect against neuronal oxidative damage induced by hydrogen peroxide [5] and oxygen-glucose deprivation [6], an in vitro model of cerebral ischemia. More recently, Rd was reported to ameliorate histological and functional outcome after focal cerebral ischemia [7-9], which is associated with mitochondrial protection and energy restoration. However, the preclinical characteristics of the neuroprotective effects of $\mathrm{Rd}$ have not been fully defined. Thus, in this study, we sought to characterize the effects of different $\mathrm{Rd}$ treatment regimes on the outcome of permanent and transient middle cerebral artery (MCA) occlusion in rats. In particular, we examined the dose response and therapeutic window of the protective effects, and we investigated whether this protection also occurs in female rats or aged rats.

\section{Methods}

\section{Focal Cerebral Ischemia}

Animal procedures were in accordance with the National Institutes (NIH) of Health Guide for the Care and Use of Laboratory Animals (NIH Publications No. 80-23, revised 1996). All efforts were made to minimize animal number and their suffering. Focal cerebral ischemia was induced by transient or permanent MCAO in Sprague-Dawley rats as described earlier [10]. Briefly, animals were anesthetized with a mixture of isoflurane (1.5-2\%), oxygen, and nitrogen. A 4-0 nylon monofilament coated with poly-Llysine was introduced through the internal carotid artery to occlude the origin of the MCA. We maintained the temperature $\left(37.0 \pm 0.5^{\circ} \mathrm{C}\right)$ using a thermostatically controlled heating blanket connected to a thermometer probe in the rectum, and at the same time we monitored physiological parameters, including blood gases $\left(\mathrm{pCO}_{2}\right.$ and $\left.\mathrm{pO}_{2}\right), \mathrm{pH}$, glucose level, and blood pressure. Blood gas was immediately analyzed using a Rapidlab 1260 system (Siemens, Erlangen, Germany). The induction of focal cerebral ischemia was verified with laser Doppler flowmetry (PeriFlux 5000; Perimed AB, Sweden), with the probe placed 6-mm lateral and 2-mm posterior to the bregma [11, 12] and secured with $\alpha$-cyanoacrylate adhesive. A drop in regional cerebral blood flow (CBF) below 30\% of baseline after the insertion of the filament was considered to be sufficient for induction of focal cerebral ischemia. Occlusion of the right
MCA was induced either permanently for $24 \mathrm{~h}$ or transiently for $2 \mathrm{~h}$ with a reperfusion period of up to 42 days. Control animals were subjected to the same surgical procedures, except that the suture was not advanced into the MCA.

\section{BBB Passage}

Rd and blood-brain barrier (BBB)-impermeable bovine serum albumin (BSA) (control) were radiolabeled with tritium and purified as previously described [13]. The radiolabeled reagents $(50 \mathrm{mg} / \mathrm{Kg})$ were injected via the tail vein of nonischemic male and female rats. After $0.5,2,8$, and $24 \mathrm{~h}$ after injection of substances, animals were terminally anesthetized with chloral hydrate and perfused carefully with saline, and the whole brain was dissected, blotted dry, and weighed. Blood was centrifuged to obtain the serum. The radioactivity was measured with a liquid scintillation counter.

\section{Administration of Rd}

Rd with a purity of $98 \%$ was obtained from Tai-He Biopharmaceutical Co. Ltd. (Guangzhou, China). Rd stock solutions were prepared in saline containing 10\% 1,3propanediol (v/v). In dose-response studies, Rd was administered at concentrations ranging from 1 to $50 \mathrm{mg} / \mathrm{kg}$. For comparison, we treated rats with PBN (N-tert-butyl-alphaphenylnitrone; Sigma, St. Louis, MO, USA) and edaravone (3-methyl-1-phenyl-2-pyrazolin-5-one; Merck, Darmstadt, Germany) using their most effective concentrations. All treatments or vehicle were applied intraperitoneally bolus 30 minutes before MCAO randomly.

The therapeutic window of $\mathrm{Rd}$ was investigated with a dose of $50 \mathrm{mg} / \mathrm{kg}$. For transient ischemia, a bolus of Rd was administered at $0,2,4$, or $8 \mathrm{~h}$ after the reperfusion. For permanent ischemia, a bolus injection of $\mathrm{Rd}$ was given at 0,2 , 4 , or $8 \mathrm{~h}$ after the onset of artery occlusion. Vehicle of a similar volume was injected $2 \mathrm{~h}$ after MCAO. Rats were assigned to each experimental group randomly before MCAO.

\section{Infarct Size Assessment}

On PODs 1, 3, and 7, animals were decapitated and 2-mm thick coronal sections from throughout the brain were stained with 2\% 2,3,5-triphenyltetrazolium chloride (TTC, Amresco Inc., Solon, OH, USA) to evaluate the infarct volume. To compensate for the effect of brain edema, the corrected infarct volume was calculated as previously described: corrected infarct area $=$ contralateral hemisphere area (nonlesioned ipsilateral area) [14]. Infarct volume was manually quantified using ImageJ (National Institutes of Health, Bethesda, MD, USA) in a blinded way and expressed as a percentage of the contralateral structure. 


\section{Measurement of Edema}

At $24 \mathrm{~h}$ after stroke, brain edema was measured by comparing the wet-to-dry tissue weight ratios as described earlier [15]. Briefly, the brain was quickly removed after the animal was killed. Then the brain was blotted to remove residual absorbent moisture and dissected through the interhemispheric fissure into the right and left hemispheres. The water content of both hemispheres was determined as the percent difference in tissue weight before and after drying for 3 days at $100^{\circ} \mathrm{C}$.

\section{Behavioral Tests}

A battery of behavioral tests, including modified neurological severity scores (mNSS) [16], modified sticky-tape test (MST) [17], and corner test [18], were performed before MCAO and at PODs 1, 3, 7, 14, 21, 28, and 42 by two investigators (X.K. and Q.Y.) who were blinded to the experimental groups. All behavioral tests were conducted in the light phase at approximately the same time each day.

\section{$m N S S$}

mNSS is a composite of motor, sensory, reflex, and balance tests. Neurological function was graded on a scale of 0 to 18 (normal score, 0; maximal deficit score, 18). In the severity scores of injury, 1 score point is awarded for the inability to perform the test or for the lack of a tested reflex; thus, the higher score, the more severe is the injury. See details in Supplemental Table 1.

\section{MST}

To assess the somatosensory dysfunction after cerebral ischemia, we performed the modified adhesive removal (sticky-tape) test. Briefly, a nonremovable tape sleeve is wrapped around the animal's paw as a tactile stimulus. When placed in a testing cage, the rats will use their mouth and to a lesser extent the other forelimb to attempt to remove the labels. The time spent attempting to remove the stimulus was recorded during a 30 -second observation period. The test was repeated on the opposite limb until sessions consist of 5 trials per limb each day for a total of 10 calculated ratios. The average ratio of left-to-right performance was calculated as the ratio of interest.

\section{Corner Test}

A rat was placed between 2 boards each with a dimension of $60 \times 40 \times 1 \mathrm{~cm}^{3}$. The edges of the 2 boards were attached at a $30^{\circ}$ angle with a small opening along the joint between the 2 boards to encourage entry into the corner. The rat was placed between the 2 angled boards facing the corner and halfway to the corner. When entering deep into the corner, both sides of the vibrissae were simultaneously stimulated. The rat reared forward and upward, then turned back to face the open end. The nonischemic rats turned either left or right, but the ischemic rats preferentially turned toward the ipsilateral (right) side. The turns in $1 v s$ the other direction were recorded from 10 trials for each rat. Turning movements that were not part of a rearing movement were not scored.

The validity and reproducibility of these behavioral tests have been tested during the preliminary study (Supplemental Table 2). For reproducibility, the intraclass correlation coefficients between the results from 2 independent examiners (X. K. and Y. Q.) are: 0.987 (MST), 0.998 (mNSS),and 0.936 (corner test), suggesting great reporting consistency between different examiners. For validity, the spearman rank-order correlation confidents between behavior test score and infarct volume on POD 3 are: $-0.81(p=0.004)$ for MST, $0.697(p=0.025)$ for $\mathrm{mNSS}$, and $0.666(p=0.036)$ for the corner test, suggesting a strong correlation between behavioral tests and histological lesion.

\section{Statistical Analysis}

All results are presented as mean with standard error of the mean. The behavioral test results were analyzed with repeated-measures of analysis of variance, followed by Tukey post hoc test. Female rat MCAO and aged rat MCAO results were analyzed using an unpaired $t$ test. Other results were analyzed with one-way analysis of variance followed by Tukey HSD post-test for multiple comparisons. Statistical significance was established at $p<0.05$.

\section{Results}

Physiologic Variables

All animals of this study showed similar values for rectal temperature, mean arterial blood pressure, arterial blood gases, and plasma glucose (Supplemental Table 3). The laser Doppler flowmetry signal showed that regional CBF was reduced equivalently in all groups during ischemia (Supplemental Fig. 1). Rats whose CBF remained $>30 \%$ of baseline were excluded from further experiment.

\section{Dose-Response Study}

A prerequisite for a direct action of Rd on the brain would be penetration of the BBB. We determined the amount of tritiated Rd in brain and serum at $0.5,2,8$, and $24 \mathrm{~h}$ after intravenous injection in noninjured rats, and calculated the 
brain/serum ratios of Rd and albumin as an index of BBB permeability. At every observation point, Rd showed a higher brain/serum ratio, which indicated passage of $\mathrm{Rd}$ through the intact BBB (Supplemental Fig. 2).

First, we investigated the dose-response characteristics of the histological and functional improvement induced by Rd. Infarct volumetry was performed on PODs 1, 3, and 7 using TTC staining (Fig. 1a). The reduction in infarct size was observed at $10 \mathrm{mg} / \mathrm{kg}$ and was greatest at $50 \mathrm{mg} / \mathrm{kg}$, whereas the $1 \mathrm{mg} / \mathrm{kg}$ dose was ineffective (Fig. 1b-d). Total infarct volume corrected for brain swelling was reduced by a mean of $59 \%, 60 \%$ and $63 \%$, respectively on PODs 1,3 and 7 after the treatment with $50 \mathrm{mg} / \mathrm{kg}$ Rd. Subanalysis showed that both cortex and striatum were significantly protected (by $57-67 \%$ and $46-55 \%$, respectively). Percentage of brain swelling was dramatically diminished by approximately $70 \%$ after treatment with $50 \mathrm{mg} / \mathrm{kg} \mathrm{Rd}$ (Supplemental Fig. 3). For comparison, we tested two other neuroprotectants: edaravone (a reactive oxygen species (ROS) scavenger approved in Japan for the treatment of cerebral infarction) [19] and PBN (an established free radical spin trap, the parent compound of NXY-059) [20]. $\mathrm{Rd}$ was significantly more effective than edaravone, and slightly more effective than PBN in reducing infarct size (Fig. 1b-d).

To determine whether the histological amelioration offered by $\mathrm{Rd}$ had consequences for the long-term postischemic behavioral changes, we evaluated neurological deficits using mNSS, MST, and corner test for as much as 6 weeks after transient ischemia (Fig. 2a-c). There were no differences in mortality among all groups (Supplemental Fig. 4). Repeated measures of analysis of variance revealed ischemic rats treated with $\mathrm{Rd} 50 \mathrm{mg} / \mathrm{kg}$ performed significantly better in all test paradigms than vehicletreated animals, whereas $10 \mathrm{mg} / \mathrm{kg}$ dose displayed benefits in MST $(p<0.001)$ and mNSS $(p=0.049)$, but not in the corner test $(p=0.603)$. Rd $50 \mathrm{mg} / \mathrm{kg}$ even induced a more robust improvement than edaravone in somatosensory dysfunction, graded according to MST $(p=0.012)$. Moreover, although body weight declined in the first week after ischemia, Rd-treated rats presented a more rapid recovery (Supplemental Fig. 5).

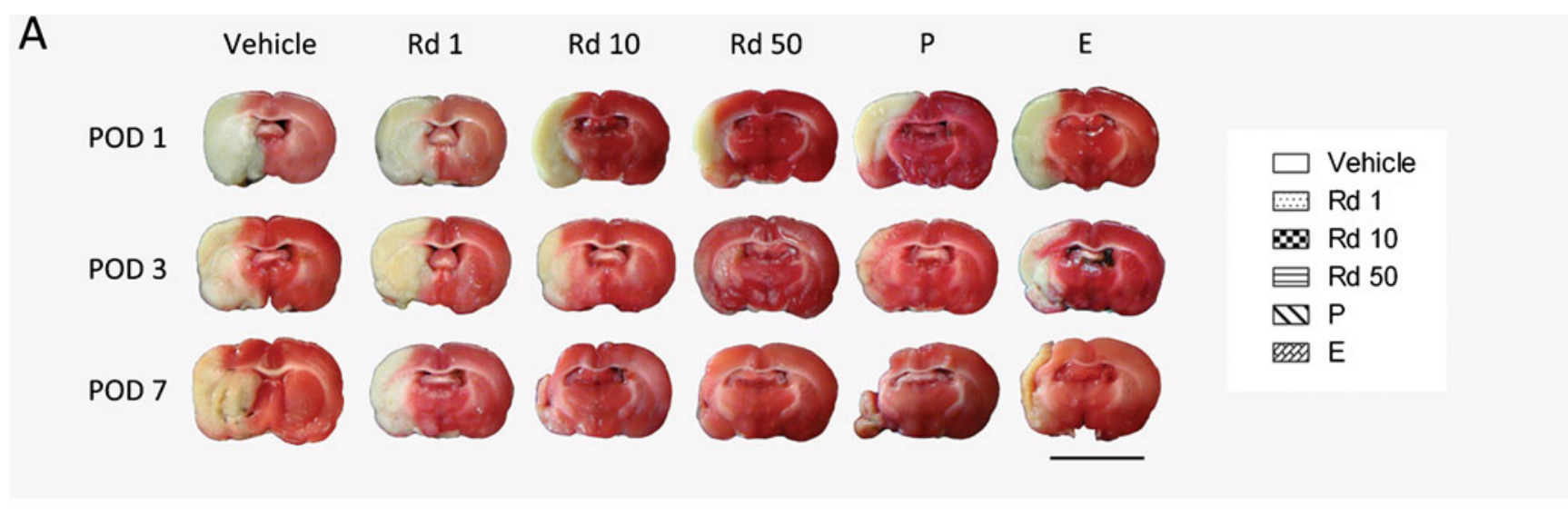

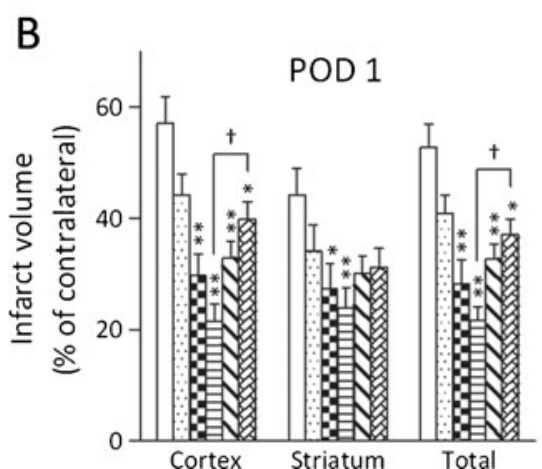

Fig. 1 Dose-response characteristics of the neuroprotection conferred by Ginsenoside Rd (Rd) in transient middle cerebral artery occlusion. (A) Representative ischemic lesions at 1.2-mm caudal from bregma in the coronal slices on postoperative days (PODs) 1, 3, and 7. Pale areas represent infarction. Treatment with $\mathrm{PBN}(\mathrm{P})$ and edaravone $(\mathrm{E})$. Scale bar, $1 \mathrm{~cm}$. Quantitative analyses of cortical, striatal, and total infarct volumes (corrected for edema) are displayed in (B-D). The infarct size
C

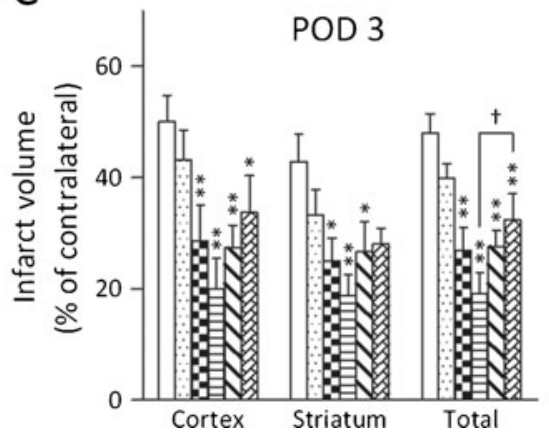

D

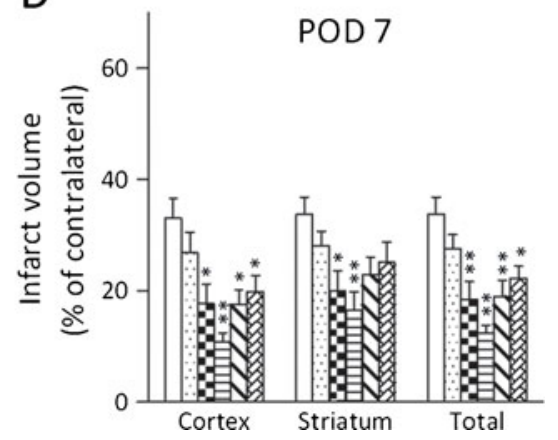

was significantly reduced in rats pretreated with 10 or $50 \mathrm{mg} / \mathrm{kg} \mathrm{Rd}$, as well as with $\mathrm{P}$ and $\mathrm{E}$, but not in rats pretreated with $1 \mathrm{mg} / \mathrm{kg} \mathrm{Rd}$, as compared with vehicle-treated counterparts. Histograms represent mean \pm standard error of the mean ( $\mathrm{n}=8$ to 9 rats for each group). ${ }^{*} p<0.05 ;{ }^{*} p<0.01 v s$ vehicle group; ${ }^{\dagger} p<0.05 v s$ edaravone group by 1 -way analysis of variance with Tukey post test 

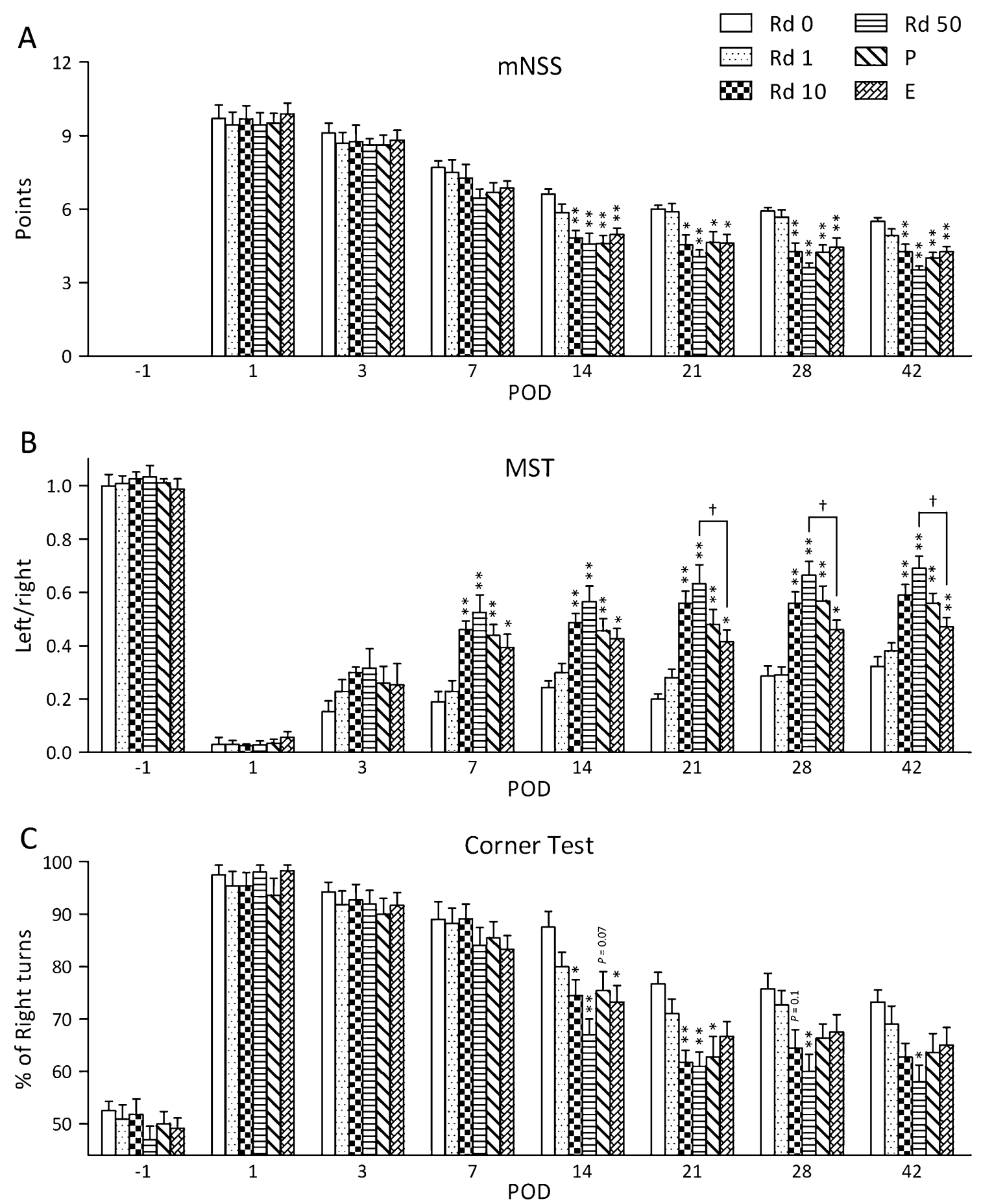

Fig. 2 Dose-response study of neurological deficit after transient middle cerebral artery occlusion. (A) Modified neurological severity scores (mNSS). (B) Modified sticky-tape test (MST). (C) Corner test. Treatment with PBN (P) and edaravone (E). Histograms represent

Therapeutic Window Study

Then we sought to define the time interval after transient ischemia at which $\mathrm{Rd}$ was still able to protect the brain $(50 \mathrm{mg} / \mathrm{kg} \mathrm{Rd}$ or vehicle was administered at $0,2,4$, or $8 \mathrm{~h}$ mean \pm standard error of the mean ( $\mathrm{n}=10$ to 12 rats for each group). ${ }^{*} p<0.05 ; * * p<0.01 v s$ vehicle group; ${ }^{\dagger} p<0.05 v s$ edaravone group by 1 -way analysis of variance with Tukey post test. $\mathrm{POD}=$ postoperative day; $\mathrm{Rd}=$ Ginsenoside $\mathrm{Rd}$

after reperfusion). Significant stroke volume reductions were observed when Rd was administered at $2 \mathrm{~h}$ (36-44\%) or $4 \mathrm{~h}(31-40 \%)$ after reperfusion, but not when the administration was delayed by $8 \mathrm{~h}$ (Fig. 3). Subanalysis showed that both cortex and striatum were significantly 


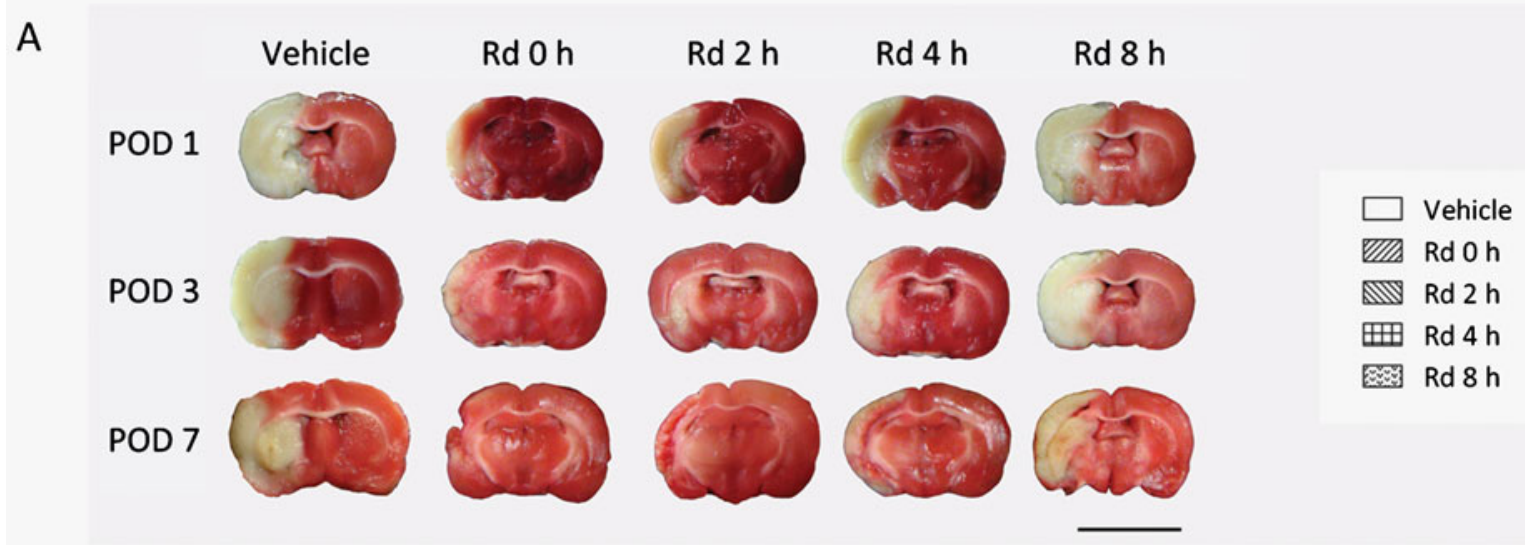

B

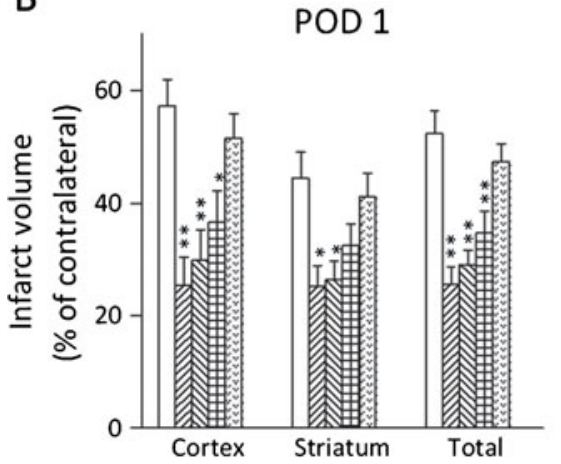

C

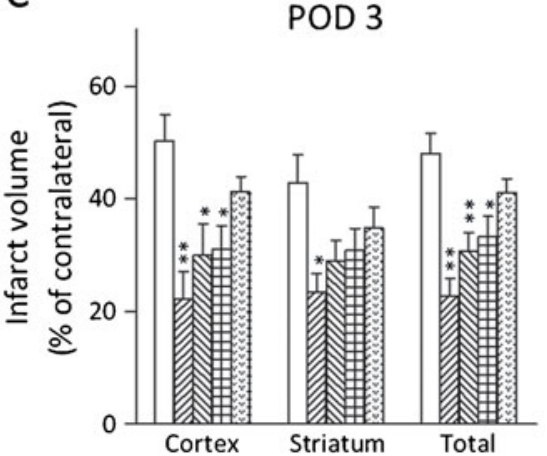

D

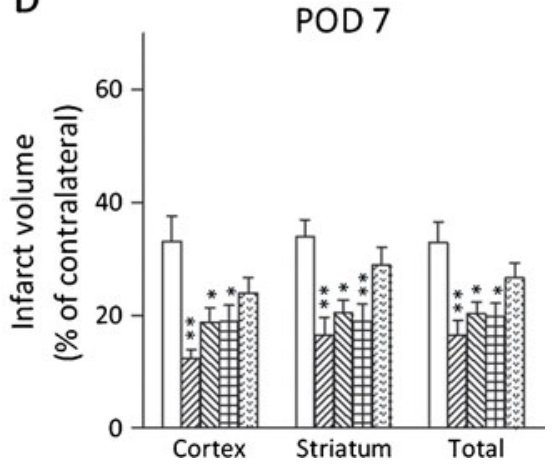

Fig. 3 Therapeutic window characteristics of Ginsenoside $\mathrm{Rd}(\mathrm{Rd})$ treatment in transient middle cerebral artery occlusion. (A) Representative ischemic lesions at $1.2-\mathrm{mm}$ caudal from bregma in the coronal slices on postoperative days (PODs) 1,3 , and 7 . Scale bar, $1 \mathrm{~cm}$. Quantitative analyses of cortical, striatal, and total infarct volumes (corrected for edema) are displayed in (B-D). The infarct size was

protected. Consistently, a significant improvement of neurological outcome was detected when Rd treatment was started 2 or $4 \mathrm{~h}$ after reperfusion as graded by mNSS (Fig. 4a), MST (Fig. 4b), and corner test (Fig. 4c).

\section{Permanent Ischemia Study}

In another model of cerebral ischemia, permanent MCAO, rats administered with $\mathrm{Rd}(50 \mathrm{mg} / \mathrm{kg})$ starting at $0,2,4$, or $8 \mathrm{~h}$ after the onset of artery occlusion. Then, 24 hours after commencing vehicle treatment, a greater damage was observed compared with that seen in the transient MCAO (62.6\% vs $52.2 \%$ of contralateral hemisphere). Treatment with $\mathrm{Rd}$ produced a significant reduction in ischemic damage when applied at 0,2 , and $4 \mathrm{~h}$ after MCAO, with neuroprotection apparent in both the cortex and the subcortex (Fig. 5). In a further group, Rd was applied starting 8-h postocclusion, whereas the mean volume of total damage was $21 \%$ smaller than the vehicle group; this decrease was not statistically significant $(p=0.212)$. significantly reduced in rats treated with $50 \mathrm{mg} / \mathrm{kg} \mathrm{Rd} 2 \mathrm{~h}$ or $4 \mathrm{~h}$ after recirculation, but not when Rd administration was delayed up to $8 \mathrm{~h}$ after recirculation, as compared with vehicle-treated counterparts. Histograms represent mean \pm standard error of the mean ( $n=8$ to 9 rats for each group). ${ }^{*} p<0.05$; ${ }^{*} p<0.01$ vs vehicle group by 1 -way analysis of variance with Tukey post test

$\mathrm{Rd}$ is Also Neuroprotective in Female Rats and Aged Rats

Many neuroprotective strategies are not effective in females or in the aged population [21, 22]. As recommended by the Stroke Therapy Academic Industry Roundtable (STAIR), efficacy studies should be further performed in females and aged animals [23, 24]. Therefore, next wesought to determine whether Rd was also neuroprotective in young adult female rats or in 16-month aged male rats. Middle cerebral artery was transiently occluded in subject rats and $\mathrm{Rd}(50 \mathrm{mg} / \mathrm{kg})$, or the vehicle was administered immediately after reperfusion. Consistent with previous studies [25], female rats had substantially smaller infarcts than male rats $(30.7 \%$ vs $52.2 \%$ of contralateral hemisphere). Figure 6a illustrated that $\mathrm{Rd}$ produced a significant reduction in ischemic damage, with an apparent neuroprotection in the cortex $(-40 \% ; p=0.02)$ and a borderline neuroprotection in the subcortical area $(-31.6 \%$; $p=0.06)$. However, Rd treatment did not improve the neurological recovery in female rats after MCAO, as measured by mNSS (Fig. 6b). Contrarily, aged rats had 

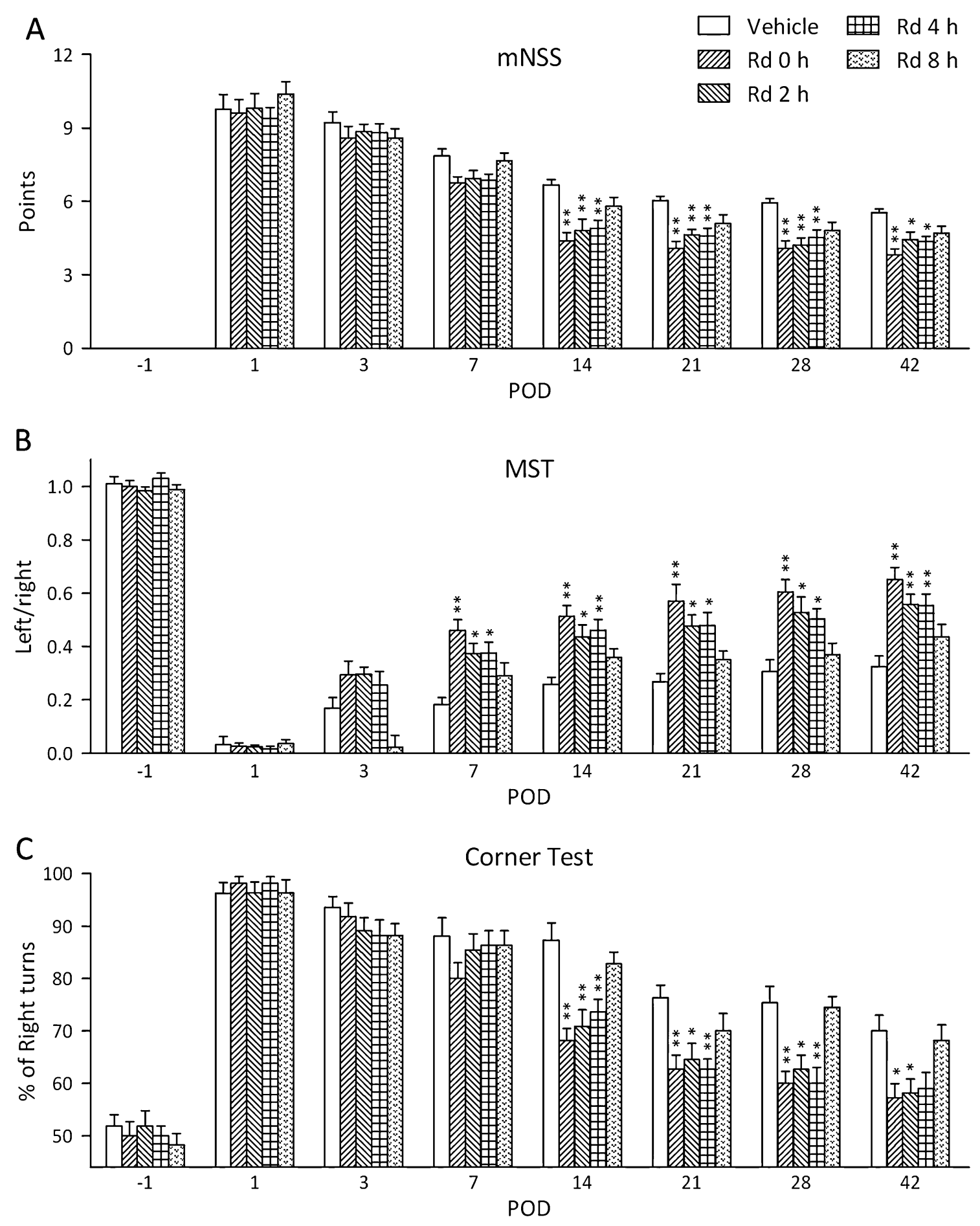

Fig. 4 Therapeutic window study of neurological deficit after transient middle cerebral artery occlusion. (A) Modified neurological severity scores (mNSS). (B) Modified sticky-tape test (MST). (C) Corner test. Histograms represent mean \pm standard error of the mean

somewhat larger infarcts than young adult rats $(58.6 \%$ vs $52.2 \%$ of contralateral hemisphere). $\mathrm{Rd}$ of $50 \mathrm{mg} / \mathrm{kg}$ resulted in an infarct volume reduction of $37 \%$ ( $p=0.005$; Fig. $6 c)$, which is correlated with behavioral improvements as graded by mNSS ( $p<0.001$; Fig. $6 \mathrm{~d})$.
( $\mathrm{n}=11$ to 12 rats for each group). ${ }^{*} p<0.05 ;{ }^{*} p<0.01 v s$ vehicle group by 1 -way analysis of variance with Tukey post-test. POD = postoperative day; $\mathrm{Rd}=$ Ginsenoside $\mathrm{Rd}$

\section{Discussion}

Here we demonstrated a dose-response relationship and defined the therapeutic window for the neuroprotective effect of $\mathrm{Rd}$ in transient and permanent cerebral ischemia. The 


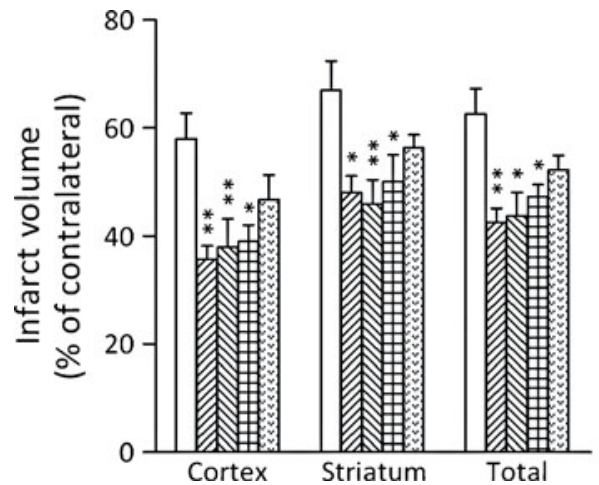

Fig. 5 The effect of delayed administration ofGinsenoside Rd (Rd) on the infarct volume after permanent middle cerebral artery occlusion (MCAO). A bolus injection of $50 \mathrm{mg} / \mathrm{kg}$ Rd was applied at 0,2 , 4, and $8 \mathrm{~h}$ after the induction of ischemia. Infarct volumetry was performed at $24-\mathrm{h}$ post-MCAO. The infarct size was significantly reduced in rats treated with $\mathrm{Rd} 2 \mathrm{~h}$ or $4 \mathrm{~h}$ after stroke, but not when Rd administration was delayed up to $8 \mathrm{~h}$ after MCAO onset, as compared with vehicletreated counterparts. Histograms represent mean \pm standard error of the mean ( $\mathrm{n}=8$ to 9 rats for each group). ${ }^{*} p<0.05 ;{ }^{* *} p<0.01 v s$ vehicle value using 1-way analysis of variance with Tukey post-test
A

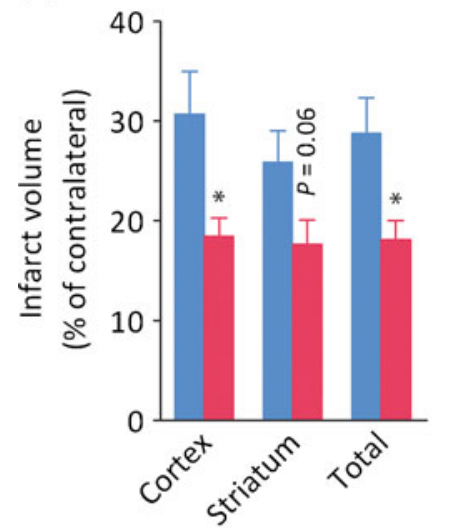

C

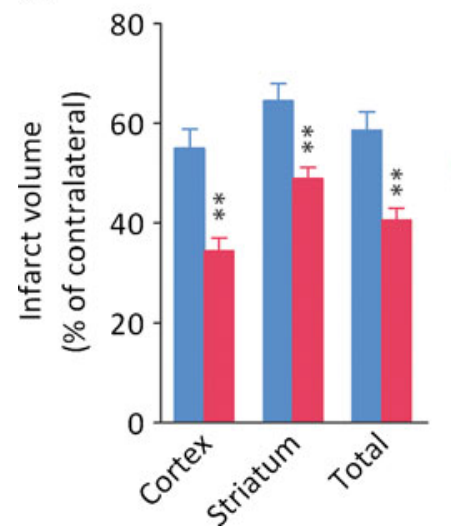

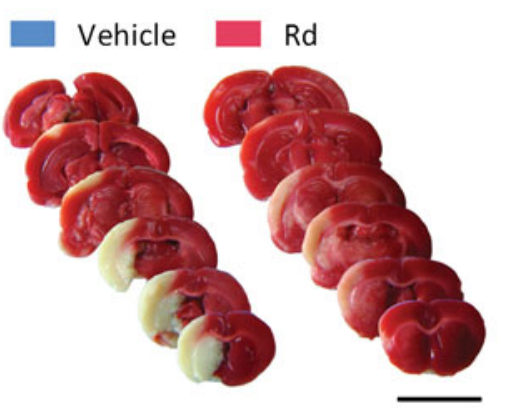

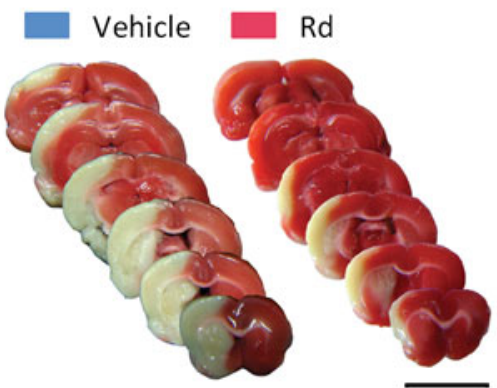

B

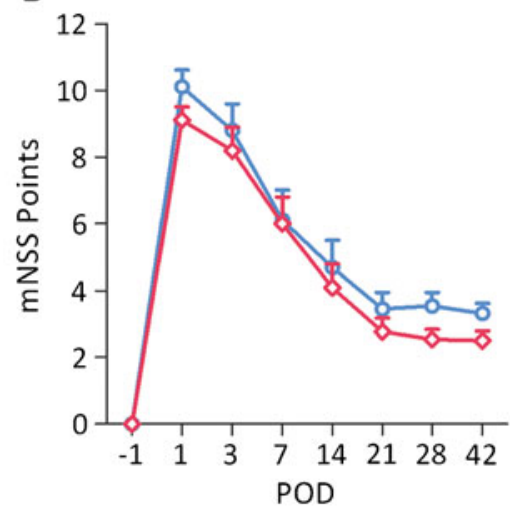

D

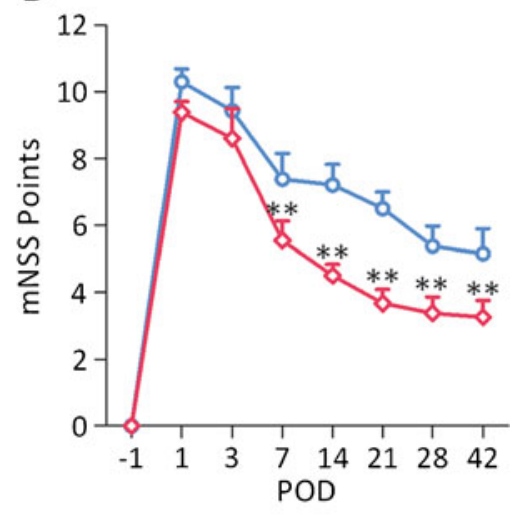

Fig. 6 The neuroprotective effect of Ginsenoside $R d(R d)$ is present in female rats and aged rats. Female or aged rats were subjected to 2 -h middle cerebral artery occlusion. A bolus injection of $50 \mathrm{mg} / \mathrm{Kg} \mathrm{Rd}$ was applied immediately after the recirculation. Infarct volumetry was performed on postoperative day (POD) 1. (A) Rd significantly reduced infarct volume in female rats. Scale bar, $1 \mathrm{~cm}$. (B) Rd did not affect the long-term neurological deficit in female rats as graded by modified neurological severity scores (mNSS). (C) Rd significantly reduced infarct volume in aged rats. Scale bar, $1 \mathrm{~cm}$. (D) Rd ameliorated the long-term neurological deficit graded by mNSS. Data represent mean \pm standard error of the mean ( $\mathrm{n}=9$ to 12 rats for each group). ${ }^{*} p<0.05 v s$ vehicle value by unpaired $t$ test 
protective effect of $\mathrm{Rd}$ is presented in moderate doses (10$50 \mathrm{mg} / \mathrm{kg}$ ) and associated with an improvement of the neurological deficits for up to 6 weeks. Importantly, $\mathrm{Rd}$ is effective, even if the treatment was delayed to $4 \mathrm{~h}$ after recirculation in transient ischemia, or to $4 \mathrm{~h}$ after artery occlusion in permanent ischemia. Furthermore, the neuroprotection also occurs in female rats and in aged rats.

In the first part of this study, we showed that the pretreatment of Rd at doses of 10 to $50 \mathrm{mg} / \mathrm{kg}$ substantially reduces the infarct size and improves long-term neurological recovery. These observations are consistent with previous reports demonstrating the neuroprotective efficacy of Rd when administered $1 \mathrm{~h}$ before ischemia at doses of 5 to $40 \mathrm{mg} / \mathrm{kg}[9,26]$. However, our infarct volumetry displays more information because of the subanalysis of total infarct volume compared with cortical and striatal infarct volume and because of the infarct volumetry performed on PODs 1, 3, and 7. The spatial pattern of the infarction suggests that $\mathrm{Rd}$ is able to salvage jeopardized tissue in both cortical and striatal areas, implying a potential role of $\mathrm{Rd}$ for white matter protection. As noted by the STAIR, it is more likely that the treatment targeting both neurons and white matter would have widespread clinical relevance [24]. Striatum is typically more severe than cortex because the blood flow in striatum is lower than in the cortex, and there is little collateral blood supply in deep white matter [27], which supports vital communication between neurons and conveys information among millions of efferent and afferent fibers. Because of its highly packed structure, lesions located in striatum can produce devastating neurological deficits, making it important that Rd significantly attenuates striatal damage.

Stroke is a highly variable clinical condition, and in particular, the delay in spontaneous reperfusion is a variant. Therefore, it is vital that potential therapeutic compounds are able to ameliorate the consequences of permanent occlusion in an animal model [23]. In contrast to transient ischemia, the free radical production was much less prominent after permanent MCAO. However, Rd at the dose of $50 \mathrm{mg} / \mathrm{kg}$, the most effective dose in transient ischemia, still produced a modest effect in a permanent occlusion model (59\% reduction of infarct size in transient MCAO vs 37\% reduction in permanent MCAO). These results indicate that $\mathrm{Rd}$ is active in a model of ischemia without reperfusion, and that this effect, therefore, is not dependent on reperfusion time, which reinforces the interest of Rd in stroke.

Another major observation made in the current investigation was the favorable time window of therapeutic opportunity that is available with Rd administration. Rodent studies appear to be relevant to address a therapeutic window for neuroprotective drugs [24]. In the past, drugs such as the N-methyl-D-aspartate antagonists demonstrated their efficacy in animals for only a limited after stroke onset; this limitation may have contributed to their lack of efficacy in clinical trials. Here we found the salutary effects of Rd were still present, even when the compound was given $4 \mathrm{~h}$ after the recirculation in transient ischemia or $4 \mathrm{~h}$ after artery occlusion in permanent ischemia. This means that it is effective in rodents in a time window that is relevant to clinical practice. However, it is worthy to note that in more than 1-half of the completed trials, the enrollment time window was beyond $6 \mathrm{~h}$ [3]. Thus, it is advised that the clinical trials of neuroprotection must be designed with a very early window to treatment. To increase the likelihood of determining efficacy, initial clinical studies of neuroprotective agents should not include patients who have received thrombolytic drugs. Potential synergistic or harmful effects when combining with thrombolytics can be explored afterward [4].

The mechanisms of the protective effects of $\mathrm{Rd}$ have been recently investigated. We have previously reported that $\mathrm{Rd}$ protects against neuronal oxidative damage induced by hydrogen peroxide [5] and oxygen-glucose deprivation [6]. More recently, we suggested that $\mathrm{Rd}$ exerts neuroprotection against transient focal ischemia in an integrated process of free radicals scavenging, mitochondrial protection, energy restoration, and apoptosis inhibition [7, 8]. Alternatively, in a model of excitotoxicity, Rd attenuates the $\mathrm{Ca}^{2+}$ overload and apoptosis produced by exposure of neuronal cultures to glutamate [28]. This effect is related by the ability of $\mathrm{Rd}$ to inhibit $\mathrm{Ca}^{2+}$ influx through receptorand store-operated $\mathrm{Ca}^{2+}$ channels without affecting voltagedependent $\mathrm{Ca}^{2+}$ entry [29]. Rd has also been noted to mitigate neuroinflammation [30] and nitric oxide overproduction [31]. Therefore, $\mathrm{Rd}$ is neuroprotective in both in vitro and in vivo models of cerebral ischemia, and could act on multiple pathways of the ischemic cascade, suggesting Rd might have a greater chance of success to impede ischemic injury in acute stroke [4].

Although many compounds with neuroprotective action are in various stages of the pharmaceutical pipeline, $\mathrm{Rd}$ represents unique advantages. Panax ginseng and its related species have already had thousands of years of human exposure with little reported toxicity. $\mathrm{Rd}$ is lipophilic and can easily diffuse across biological membranes and BBBs, a feature that contrasts strongly with NXY-059, which has a very low BBB permeability and negligible cellular penetration [3]. Plus, unlike some perfusion-dependent neuroprotective therapies, such as tirilazad [32], hyperbaric oxygenation [33], and anti-intercellular adhesion molecule-1 antibody [34] are efficacious only in transient cerebral ischemia models. Rd provides substantial neuroprotection in both transient and permanent MCAO models of stroke. Moreover, we herein compared the neuroprotective potency of Rd with other established antioxidants with 
neuroprotective abilities. Rd resulted in better histological and functional improvement than edaravone, anROS scavenger approved in Japan for the treatment of cerebral infarction.

Despite spectacular success of neuroprotective therapy in an animal model of stroke, the translation of neuroprotective benefits from bench to bedside has not been successful. Past failures enhance, not diminish, the need for future neuroprotection research. It is hoped that adherence to the STAIR recommendations may enhance the chances for success [24]. In the present study, all efforts were implemented with the intent to better the study quality, according to STAIR criteria, to ensure the results were reliable and not biased. Currently $\mathrm{Rd}$ is being developed to treat patients with acute ischemic stroke. Several studies have been reported demonstrating the pharmacokinetics [35], safety [36], and preliminary efficacy [37] of Rd in human subjects. More recently, a phase III multicenter clinical trial of $\mathrm{Rd}$ showed a benefit in terms of the prespecified primary endpoint, the distribution of disability as measured by the modified Rankin score at 90 days after stroke onset. Together, both laboratory and clinical evidence indicate that $\mathrm{Rd}$ may be a promising neuroprotectant and provide support for future studies to confirm whether $\mathrm{Rd}$ is beneficial in ischemic stroke.

In summary, the present study suggested that $\mathrm{Rd}$ treatment reduces both cerebral lesions and functional deficit induced by focal cerebral ischemia. We demonstrated that this effect occurs within a large therapeutic window and is reproducible in different models of stroke (with or without reperfusion) and different populations (male or female, young adult or aged). Therefore, Rd appears to be worthy of clinical development as a treatment for acute ischemic stroke.

Acknowledgments This study was supported in part by the National Natural Science Foundation of China (Grant No. 81073094). Ruidong Ye received Post-graduate Study Abroad Scholarship from China Scholarship Council. The authors are grateful to Tai-He Biopharmaceutical Co. Ltd. for supplying ginsenoside Rd. We also thank Shiquan Wang for his technical assistance with MCAO model experiments. Full conflict of interest disclosure is available in the electronic supplementary material for this article.

Disclosure The authors declare no conflict of interest.

\section{References}

1. Sacco RL, Chong JY, Prabhakaran S, Elkind MS. Experimental treatments for acute ischaemic stroke. Lancet 2007;369:331-341.

2. Gropen TI, Gagliano PJ, Blake CA, et al. Quality improvement in acute stroke: The New York State Stroke Center Designation Project. Neurology 2006;67:88-93.

3. Ginsberg MD. Neuroprotection for ischemic stroke: Past, present and future. Neuropharmacology 2008;55:363-389.
4. Savitz SI, Fisher M. Future of neuroprotection for acute stroke: In the aftermath of the Saint trials. Ann Neurol 2007;61:396-402.

5. Ye R, Han J, Kong X, et al. Protective effects of ginsenoside Rd on pc12 cells against hydrogen peroxide. Biol Pharm Bull 2008;31:1923-1927.

6. Ye R, Li N, Han J, et al. Neuroprotective effects of ginsenoside $\mathrm{Rd}$ against oxygen-glucose deprivation in cultured hippocampal neurons. Neurosci Res 2009;64:306-310.

7. Ye R, Zhang X, Kong X, et al. Ginsenoside Rd attenuates mitochondrial dysfunction and sequential apoptosis after transient focal ischemia. Neuroscience 2011;178:169-180.

8. Ye R, Yang Q, Kong X, et al. Ginsenoside Rd attenuates early oxidative damage and sequential inflammatory response after transient focal ischemia in rats. Neurochem Int 2011;58:391-398.

9. Lu Y, Peng N, Lu Z, Sang H, Yang Q, Xiong L. Neuroprotective effect of ginsenoside Rd predose on brain in rats. Chin $\mathrm{J}$ Neurosurg Dis Res 2009;8:496-499.

10. Belayev L, Alonso OF, Busto R, Zhao W, Ginsberg MD. Middle cerebral artery occlusion in the rat by intraluminal suture. Neurological and pathological evaluation of an improved model. Stroke 1996;27:1616-1623.

11. Toung TJ, Bhardwaj A, Dawson VL, Dawson TM, Traystman RJ, Hurn PD. Neuroprotective fk506 does not alter in vivo nitric oxide production during ischemia and early reperfusion in rats. Stroke 1999;30:1279-1285.

12. Rusa R, Alkayed NJ, Crain BJ, et al. 17beta-estradiol reduces stroke injury in estrogen-deficient female animals. Stroke 1999;30:1665-1670.

13. Schneider A, Kruger C, Steigleder T, et al. The hematopoietic factor g-csf is a neuronal ligand that counteracts programmed cell death and drives neurogenesis. J Clin Invest 2005;115:20832098.

14. Lin TN, He YY, Wu G, Khan M, Hsu CY. Effect of brain edema on infarct volume in a focal cerebral ischemia model in rats. Stroke 1993;24:117-121.

15. Nishi H, Watanabe T, Sakurai H, Yuki S, Ishibashi A. Effect of mci-186 on brain edema in rats. Stroke 1989;20:1236-1240.

16. Chen J, Li Y, Wang L, et al. Therapeutic benefit of intravenous administration of bone marrow stromal cells after cerebral ischemia in rats. Stroke 2001;32:1005-1011.

17. Komotar RJ, Kim GH, Sughrue ME, et al. Neurologic assessment of somatosensory dysfunction following an experimental rodent model of cerebral ischemia. Nat Protoc 2007;2:2345-2347.

18. Shen LH, Li Y, Chen J, et al. Intracarotid transplantation of bone marrow stromal cells increases axon-myelin remodeling after stroke. Neuroscience 2006;137:393-399.

19. Yoshida H, Yanai H, Namiki Y, Fukatsu-Sasaki K, Furutani N, Tada N. Neuroprotective effects of edaravone: A novel free radical scavenger in cerebrovascular injury. CNS Drug Rev 2006;12:920.

20. Floyd RA. Serendipitous findings while researching oxygen free radicals. Free Radic Biol Med 2009;46:1004-1013.

21. Popa-Wagner A, Carmichael ST, Kokaia Z, Kessler C, Walker LC. The response of the aged brain to stroke: too much, too soon? Curr Neurovasc Res 2007;4:216-227.

22. Hurn PD, Vannucci SJ, Hagberg H. Adult or perinatal brain injury: does sex matter? Stroke 2005;36:193-195.

23. Stroke Therapy Academic Industry Roundtable. Recommendations for standards regarding preclinical neuroprotective and restorative drug development. Stroke 1999;30:2752-2758.

24. Fisher M, Feuerstein G, Howells DW, et al. Update of the stroke therapy academic industry roundtable preclinical recommendations. Stroke 2009;40:2244-2250.

25. Liu F, Yuan R, Benashski SE, McCullough LD. Changes in experimental stroke outcome across the life span. J Cereb Blood Flow Metab 2009;29:792-802. 
26. Yuan L, Dong H, Zhang H, Qu Y, Xiong L. Neuroprotective effect of ginsenoside $\mathrm{Rd}$ and its combined drug treatment. Chin $\mathrm{J}$ Neurosurg Dis Res 2010;9:254-257.

27. Lo EH, Dalkara T, Moskowitz MA. Mechanisms, challenges and opportunities in stroke. Nat Rev Neurosci 2003;4:399-415.

28. Li XY, Liang J, Tang YB, Zhou JG, Guan YY. Ginsenoside Rd prevents glutamate-induced apoptosis in rat cortical neurons. Clin Exp Pharmacol Physiol 2010;37:199-204.

29. Guan YY, Zhou JG, Zhang Z, et al. Ginsenoside-rd from panax notoginseng blocks ca2+ influx through receptor- and storeoperated ca2+ channels in vascular smooth muscle cells. Eur $\mathrm{J}$ Pharmacol 2006;548:129-136.

30. Lin WM, Zhang YM, Moldzio R, Rausch WD. Ginsenoside Rd attenuates neuroinflammation of dopaminergic cells in culture. $\mathrm{J}$ Neural Transm Suppl 2007;72:105-112.

31. Choi SS, Lee JK, Han EJ, et al. Effect of ginsenoside Rd on nitric oxide system induced by lipopolysaccharide plus tnfalpha in c6 rat glioma cells. Arch Pharm Res 2003;26:375382.
32. Xue D, Slivka A, Buchan AM. Tirilazad reduces cortical infarction after transient but not permanent focal cerebral ischemia in rats. Stroke 1992;23:894-899.

33. Lou M, Eschenfelder CC, Herdegen T, Brecht S, Deuschl G. Therapeutic window for use of hyperbaric oxygenation in focal transient ischemia in rats. Stroke 2004;35:578-583.

34. Zhang RL, Chopp M, Jiang N, et al. Anti-intercellular adhesion molecule-1 antibody reduces ischemic cell damage after transient but not permanent middle cerebral artery occlusion in the wistar rat. Stroke 1995;26:1438-1443.

35. Yang L, Deng $Y, X u ~ S$, Zeng X. In vivo pharmacokinetic and metabolism studies of ginsenoside rd. J Chromatogr B Analyt Technol Biomed Life Sci 2007;854:77-84.

36. Zeng X, Deng Y, Feng Y, et al. Pharmacokinetics and safety of ginsenoside $\mathrm{Rd}$ following a single or multiple intravenous dose in healthy chinese volunteers. J Clin Pharmacol 2010;50:285-292.

37. Liu X, Xia J, Wang L, et al. Efficacy and safety of ginsenoside-rd for acute ischaemic stroke: a randomized, double-blind, placebocontrolled, phase II multicenter trial. Eur J Neurol 2009;16:569-575. 